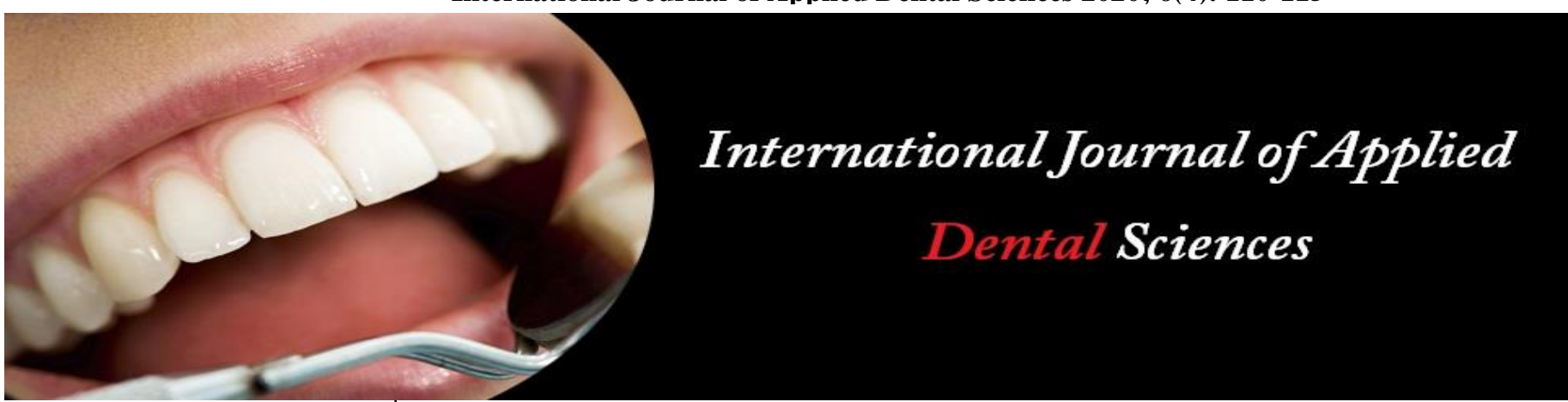

ISSN Print: 2394-7489

ISSN Online: 2394-7497

IJADS 2020; 6(4): 110-115

(C) 2020 IJADS

www.oraljournal.com

Received: 25-08-2020

Accepted: 27-09-2020

Dr. Liberia D'souza

BDS Post-Graduate Student

Department of Periodontics,

Goa Dental College \& Hospital,

Bambolim, Goa, India

Dr. Sandeep Anant Lawande

MDS Assistant Professor

Department of Periodontics,

Goa Dental College \& Hospital,

Bambolim, Goa, India

Dr. James Samuel

MDS Professor Department of

Periodontics, Goa Dental College

\& Hospital, Bambolim, Goa,

India
Corresponding Author:

Dr. Sandeep Anant Lawande MDS Assistant Professor

Department of Periodontics, Goa Dental College \& Hospital,

Bambolim, Goa, India

\section{Management of ankyloglossia using electrosurgery: A case series}

\author{
Liberia D'souza, Sandeep Anant Lawande and James Samuel
}

DOI: https://doi.org/10.22271/oral.2020.v6.i4b.1055

\section{Abstract}

Ankyloglossia, or tongue tie, is a congenital oral anomaly with short, tight and thick lingual frenum which may restrict mobility of tongue and may subsequently lead to a range of problems such as difficulties in breast feeding during infancy, difficulty in mastication, speech impairment, poor oral hygiene, malocclusion, behaviour problems during childhood, adolescence and even adulthood. In this case series, four adult patients with ankyloglossia were treated by performing lingual frenectomy using electrosurgery followed by post-operative recall and speech therapy. A marked improvement in tongue movement and speech was observed at follow-up visits in all the treated cases. Electrosurgery proved to be a safe, reliable, effective and patient-friendly alternative treatment option for ankyloglossia.

Keywords: Ankyloglossia, lingual frenectomy, electrosurgery

\section{Introduction}

Lingual frenulum is the thin strip of tissue that runs vertically from the floor of the mouth to the ventral surface of the tongue. When the frenum is tight and thick and/or its place of insertion limits the mobility of the tongue, it can result in ankyloglossia (commonly known as 'tongue-tie') ${ }^{[1]}$. Etymologically, the term "ankyloglossia" originates from the Greek words "agkilos" (curved) and "glossa" (tongue) ${ }^{[2]}$.

The prevalence of ankyloglossia varies from $0.1 \%$ to $10.7 \%$, being higher in neonates $(1.72 \%$ to $10.7 \%)$ than in adults $(0.1 \% \text { to } 2.08 \%)^{[3,5]}$. The occurrence of this anomaly is three times more common in males than females, without any racial predilection ${ }^{[6]}$.

Ankyloglossia varies in degree of severity from mild cases characterized by mucous membrane bands to complete ankyloglossia whereby the tongue is tethered to the floor of the mouth ${ }^{[7]}$. It results in several consequences ranging from difficulties in breastfeeding during infancy to difficulty in mastication, speech difficulties in pronunciation of consonants, poor oral hygiene, gingival recession on the lingual surface of mandibular anterior teeth, malocclusion, salivary profusion and difficulty in stability of mandibular prosthesis in adults. The cumulative effect of these barriers often has negative impact on self-esteem and confidence, and emotional or behavioural problems during childhood, adolescence and even adulthood ${ }^{[3,8]}$.

In many individuals, ankyloglossia is asymptomatic; the condition may resolve spontaneously as growth progresses or affected individuals may learn to compensate adequately for their decreased lingual mobility ${ }^{[9]}$. The etiopathogenesis of ankyloglossia is unknown; it can occur either as a sole anomaly in the vast majority of cases or in association with other craniofacial abnormalities ${ }^{[10]}$.

Various techniques have been employed for correction of ankyloglossia including conventional scalpel technique, electrosurgery and lasers. There is no consensus about the optimal timing, indication and type of surgical intervention for ankyloglossia. Postsurgical tongue exercises and speech therapy are necessary to achieve satisfactory results ${ }^{[7,10]}$.

\section{Clinical Assessment of Ankyloglossia}

Tongue mobility, appearance and speech difficulty

Tongue mobility and appearance associated with the insertion, as well as attachment and shortness of the lingual frenum should be evaluated. 
Furthermore, instances of speech difficulty resulting from the limited tongue movements can be checked by vocalizing some letters and words (sounds such as "s", " $z$ ", "t", "d", "l", "zh", "ch", "th", "r" and words like "ta", "te", time, water, cat, etc. ${ }^{[10]}$.

\section{Kotlow's classification of ankyloglossia}

Assessment of lingual frenum should be done based on measurement of free tongue (defined as length of the tongue from the insertion of lingual frenum into the base of the tongue to the tip of the tongue) (Fig. 1) using Kotlow's classification as follows ${ }^{[2]}$.

Table 1: Kotlow's classification

\begin{tabular}{|c|c|}
\hline Type of ankyloglossia & Length of free tongue \\
\hline Clinically acceptable & $>16 \mathrm{~mm}$ \\
\hline Class I (Mild) & $12-16 \mathrm{~mm}$ \\
\hline Class II (Moderate) & $8-11 \mathrm{~mm}$ \\
\hline Class III (Severe) & $3-7 \mathrm{~mm}$ \\
\hline Class IV (Complete) & $<3 \mathrm{~mm}$ \\
\hline
\end{tabular}

Class III and class IV categories should be given special consideration because they severely restrict the tongue movement ${ }^{[7]}$.

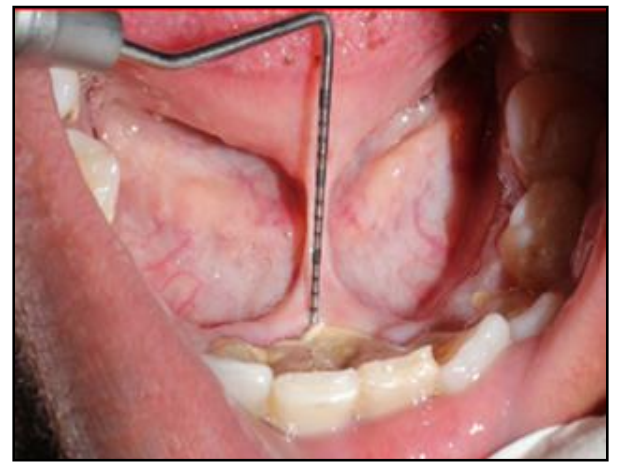

Fig 1: Length of free tongue

\section{Case Series}

The present case series describes four cases of ankyloglossia which had reported to the Department of Periodontics, Goa Dental College \& Hospital, Bambolim, Goa. Lingual frenectomy was performed in all cases using electrosurgical unit (Carlo de Giorgi, Milan, Italy) (Fig. 2). Informed consent was obtained for the same.

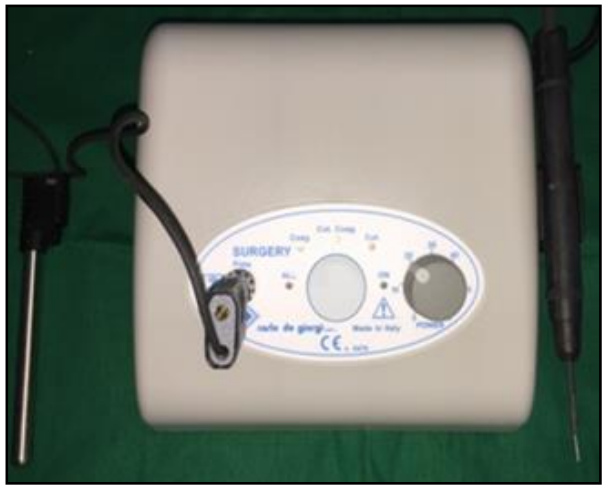

Fig 2: Electrosurgery unit

\section{Case 1}

A 25 year old systemically healthy male patient presented with the complaint of difficulty in speech and history of speech problems and restricted tongue movements since childhood. Intraoral examination revealed restricted tongue movements, bifid appearance of the tip of the tongue during protrusion (Fig. 3), class III ankyloglossia (Kotlow's classification) (Fig. 4, 5), poor oral hygiene particularly in the mandibular lingual anterior region, short and thick lingual frenum with presence of 2-3 $\mathrm{mm}$ lingual gingival recession in relation to 31 and 41 and mild crowding of the mandibular anterior teeth (Fig. 4, 5). Supragingival and subgingival scaling and root planing was performed followed by lingual frenectomy using electrosurgery.

The surgical area was anesthetized using $2 \%$ lignocaine with 1:80,000 adrenaline. Lingual frenum was held with the hemostat at the depth of vestibule. With optimal settings on the electrosurgery unit, two incisions were placed along superior and inferior aspects of hemostat with loop electrode using gentle brushing strokes (Fig. 6). The frenulum was then removed, leaving a diamond-shaped wound (Fig. 7). Fiber remnants were excised and a blunt dissection was performed. The ablated tissue was continuously mopped using wet gauze piece to take care of the charred tissue and prevent excessive thermal damage to underlying soft tissue; the attachment of frenum to the alveolar ridge was also excised to prevent tension on the gingiva. Immediate postoperative view showed arrested bleeding and no requisite for sutures (Fig.7). Protrusive tongue movement was checked. Analgesics (ibuprofen $400 \mathrm{mg}$ ), 0.2\% chlorhexidine mouthwash (Hexidine, ICPA, India) and warm saline rinses were prescribed. Patient was also advised to perform tongue exercises. 3 weeks post operatively, the patient was referred for speech therapy.

One week postoperative view showed presence of slough in the operated site indicating healing process (Fig. 8). Clinically, complete healing along with increased tongue movement was observed after 1, 3 and 6 months follow-up (Fig 9, 10). Oral hygiene maintenance in relation to lingual aspects of mandibular anterior teeth was remarkably facilitated postoperatively. Speech difficulties were significantly improved following speech therapy.

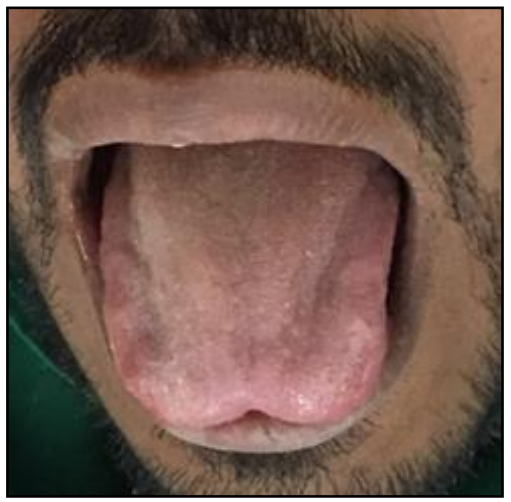

Fig 3: (Case 1) Pre-operative view (tongue protrusion)

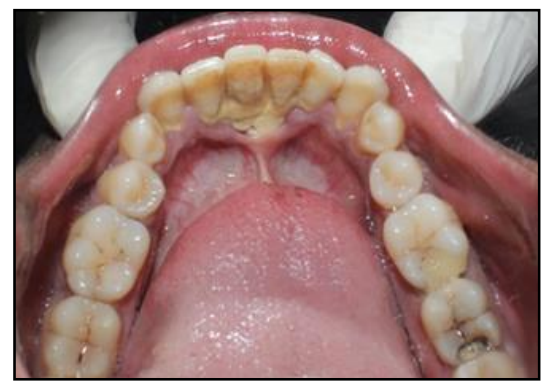

Fig 4: (Case 1) Pre-operative view (before scaling) 


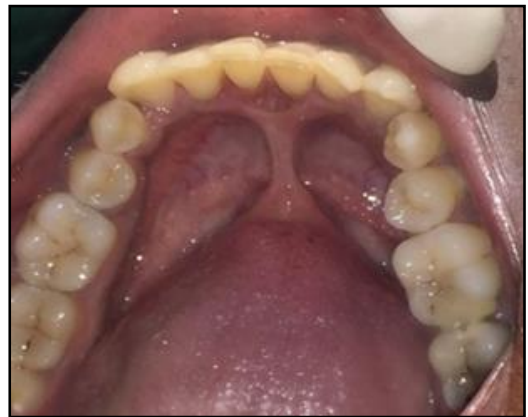

Fig 5: (Case 1) Pre-operative view (after scaling)

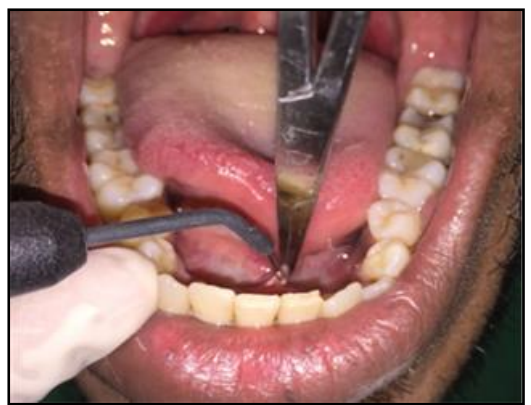

Fig 6: (Case 1) Use of hemostat and electrode

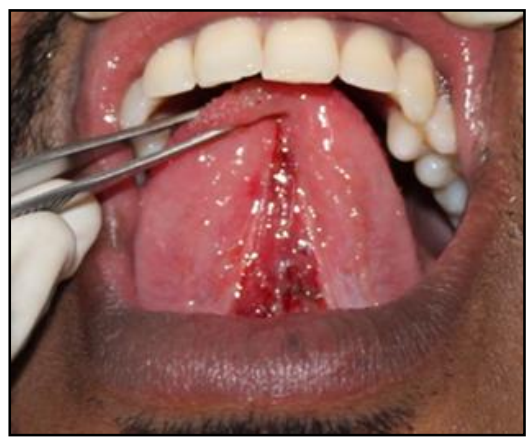

Fig 7: (Case 1) Excision of lingual frenum

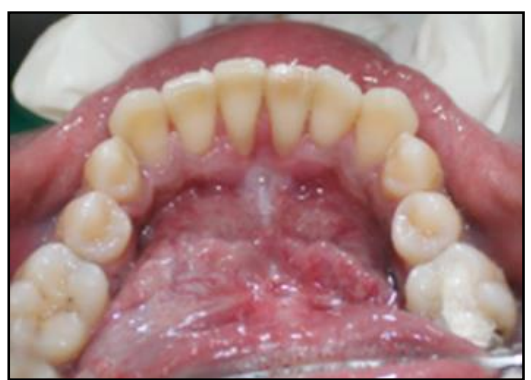

Fig 8: (Case 1) One week post-operative view

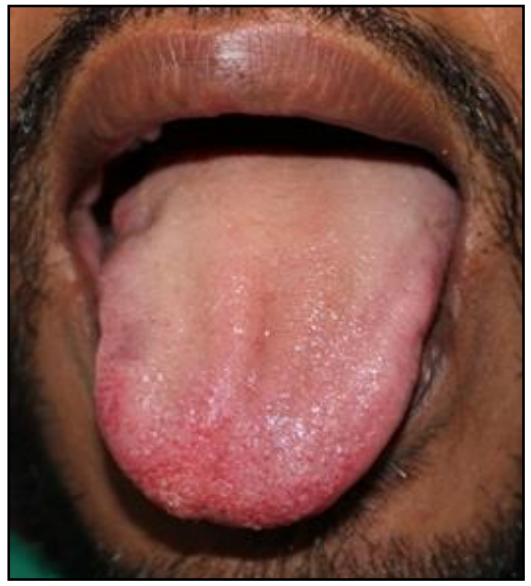

Fig 9: (Case 1) Post-operative view (tongue protrusion)

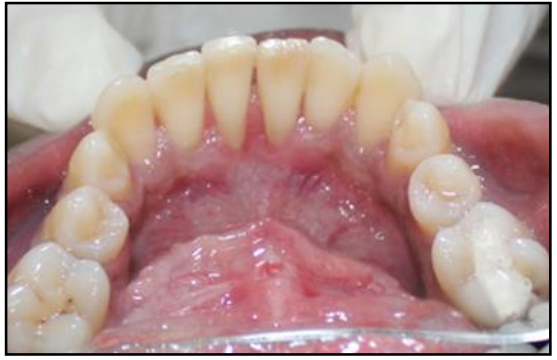

Fig 10: (Case 1) Six months post-operative view

Case 2

A systemically healthy male aged 21 years presented with complaint of difficulty in speech and protrusion of tongue. Intraoral examination revealed Kotlow's class III ankyloglossia, mild crowding in relation to 31 and 41, protrusion of the tongue only to the lower lip and not beyond that (Figs. 11, 12).

After routine scaling, lingual frenectomy was performed under local anesthesia using electrosurgical method. Postoperative wound healing was uneventful. (Figs. 13, 14) Patient was referred for speech therapy 3 weeks postoperatively.

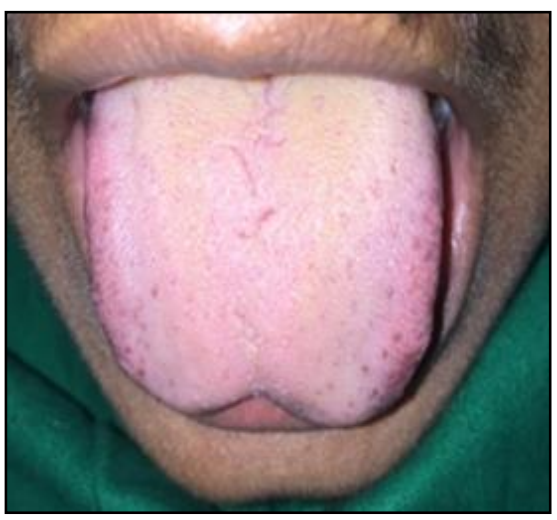

Fig 11: (Case 2) Pre-operative view (tongue protrusion)

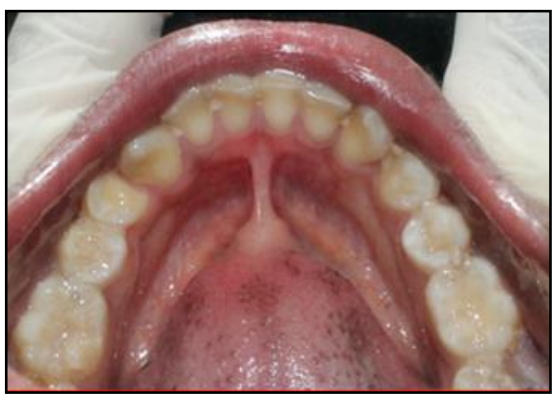

Fig 12: (Case 2) Pre-operative view

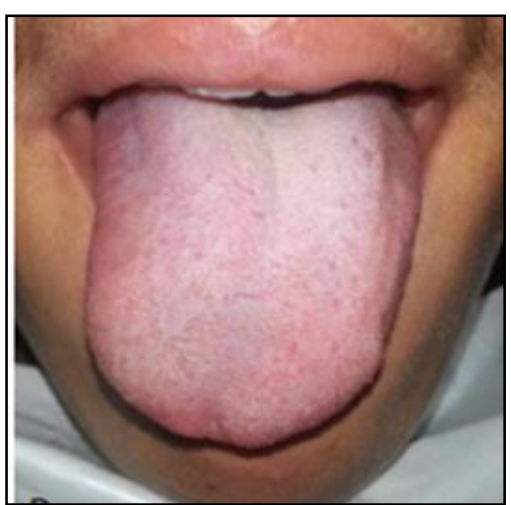

Fig 13: (Case 2) Post-operative view (tongue protrusion) 


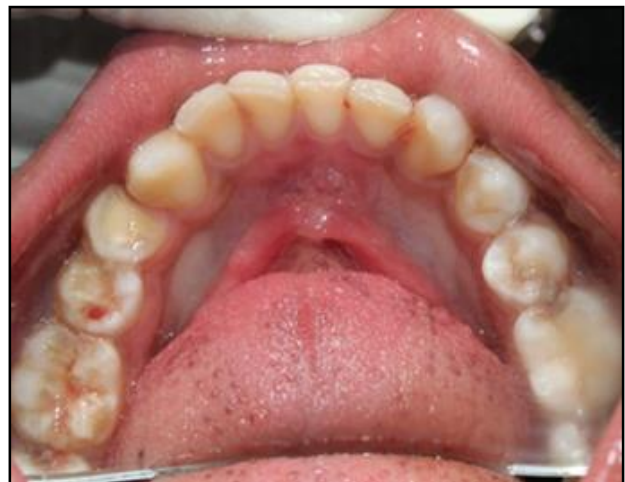

Fig 14: (Case 2) Six months post-operative view

\section{Case 3}

A 15 year old systemically healthy male patient reported with a complaint of difficulty in protrusion of tongue. Intraoral examination revealed Kotlow's class III ankyloglossia, spacing between 31 and 41, difficulty in protrusion of the tongue beyond the lower lip (Figs. 15, 16).

Post-scaling, lingual frenectomy was planned and performed under local anesthesia using electrosurgical method. Postoperative wound healing was uneventful following which speech therapy was commenced (Figs. 17, 18).

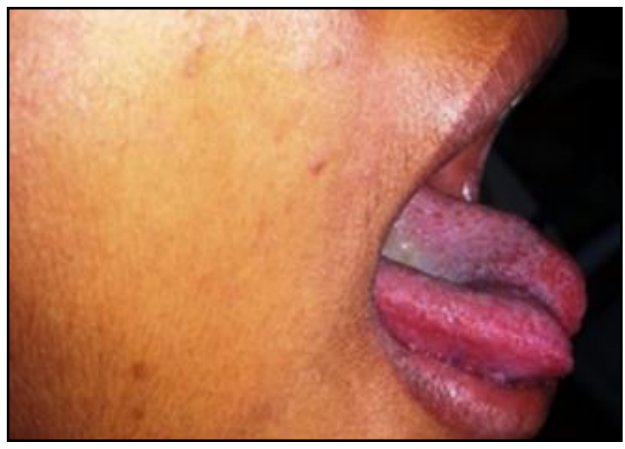

Fig 15: (Case 3) Pre-operative view (tongue protrusion)

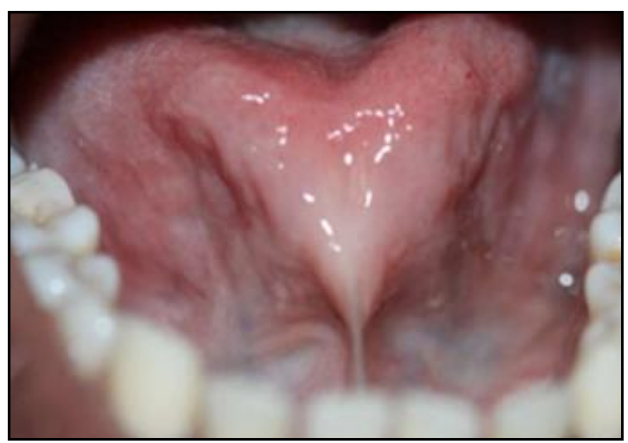

Fig 16: (Case 3) Pre-operative view

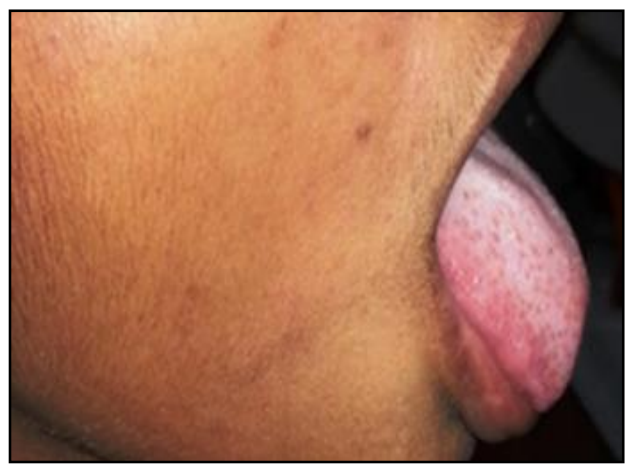

Fig 17: (Case 3) Post-operative view (tongue protrusion)

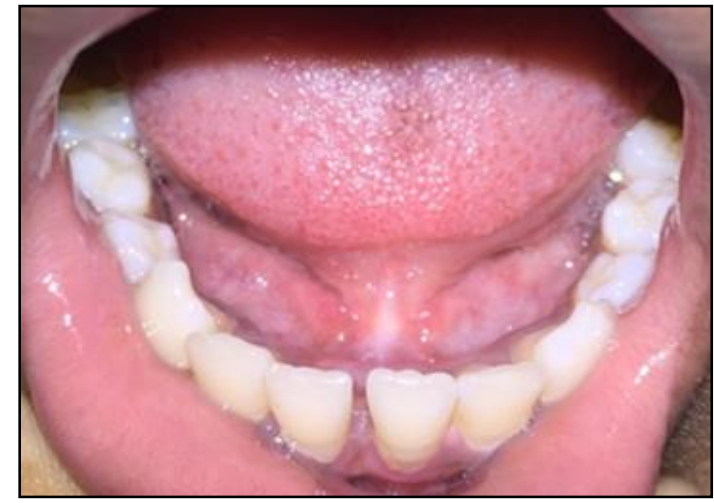

Fig 18: (Case 3) Six months post-operative view

\section{Case 4}

A systemically healthy female aged 54 years was referred from the Department of Prosthodontics for lingual frenectomy for the purpose of fabrication of cast partial denture in relation to missing posterior teeth. Intraoral examination revealed Kotlow's class III ankyloglossia with mild speech difficulty (Figs. 19, 20).

After routine scaling, lingual frenectomy was performed under local anesthesia using electrosurgery. Post-operative wound healing was uneventful (Figs. 21, 22). Speech therapy was carried out by the speech therapist and the patient was then referred for prosthodontic rehabilitation.

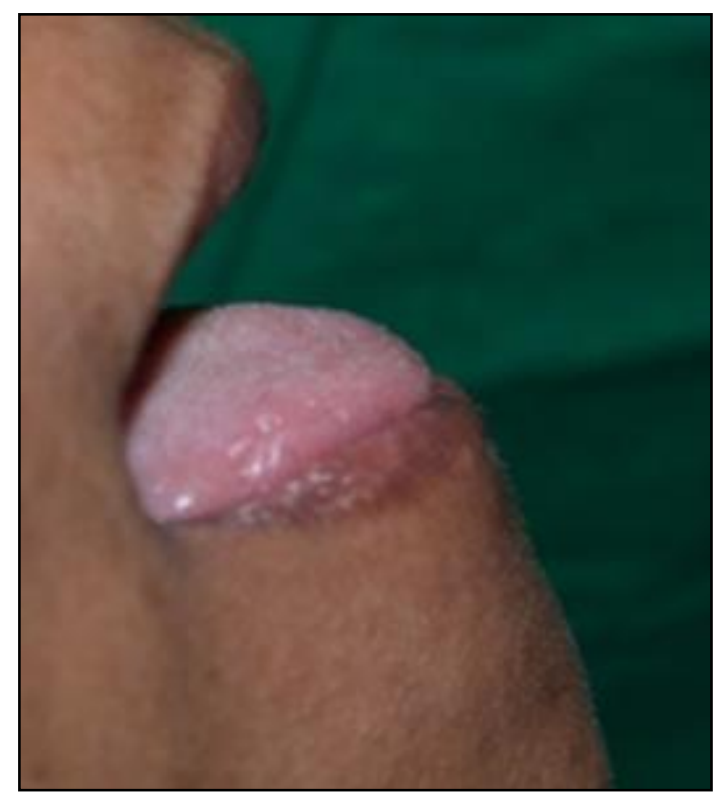

Fig 19: (Case 4) Pre-operative view (tongue protrusion)

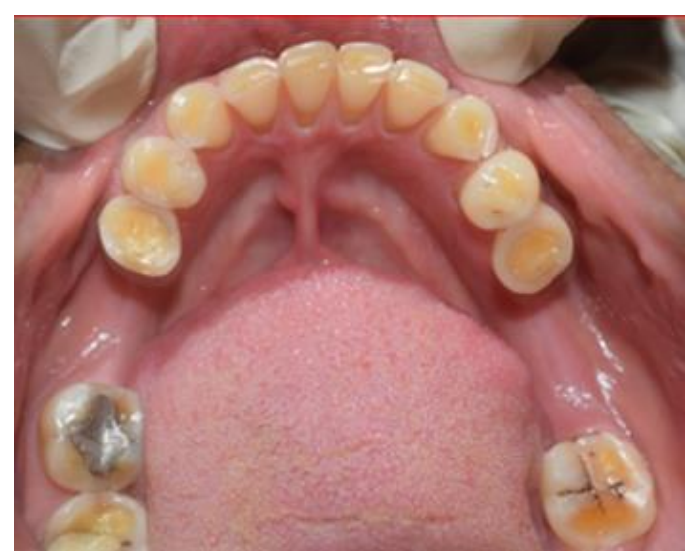

Fig 20: (Case 4) Pre-operative view 


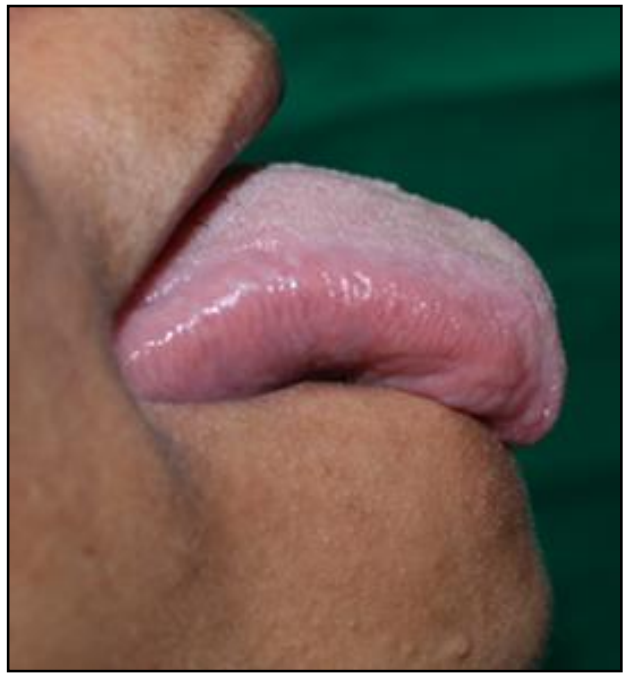

Fig 21: (Case 4) Post-operative view (tongue protrusion)

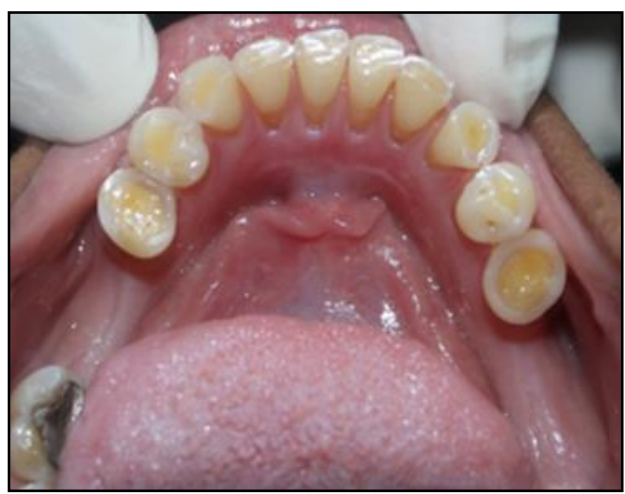

Fig 22: (Case 4) Six months post-operative view

\section{Discussion}

Proper management of ankyloglossia includes timely and appropriate surgical intervention, followed by speech therapy. The correction of ankyloglossia can be done at any age depending upon the patient's history of speech, mechanical and social difficulties ${ }^{[11]}$. However, correction at an early age reduces the risk of latent complications ${ }^{[10]}$.

In the present case series, all four cases of ankyloglossia were subjected to surgical correction by frenectomy procedure using electrosurgery. Frenectomy means surgical excision of frenum involving complete removal of the frenum ${ }^{[12]}$.

Electrosurgery involves the intentional passage of highfrequency waveforms or currents, through the tissues of the body to achieve a controllable surgical effect ${ }^{[13]}$. By varying the mode of application of this type of current, the clinician can use electrosurgery for cutting or coagulating soft tissues [14]. Appropriate operation guidelines as well as precautions need to be strictly followed and were accordingly followed as regards to the present case series.

In conventional surgical technique using the scalpel, hemostasis is difficult to achieve, surgical site visibility is inadequate with increased possibility of damage to vital structures. Using electrosurgery for the present cases offered the advantagesof minimal time consumption, reduced operator fatigue, hemostasis while cutting with no requirement of sutures. Less bleeding during the procedure helps in improving visibility to the surgical site and enhances tissue manipulation. Patients who undergo conventional frenectomy procedures using a scalpel often experience postsurgical pain and discomfort, which might be attributed to the fact that it is a more intrusive surgical procedure involving blood loss, wide surgical wound and suturing. The sutures also contribute to the discomfort postoperatively since they interfere with regular functions such as speech and intake of food. Need to do suturing was eliminated while treating the patient with electrosurgery, which also reduced the risk of post-operative infection ${ }^{[3,9,12]}$.

The advantages of frenectomy with electrosurgery have been compared to that of frenectomy with laser and have been found to be similar ${ }^{[6,10]}$. However, the main disadvantage of laser is that it is much more expensive than an electrosurgical unit ${ }^{[6]}$. It has been reported that, the speed of incisions and excisions, measured in seconds, was also faster for electrosurgery unit as compared to laser. The collateral tissue damage was also less in electrosurgery group as compared to laser. Other advantages of electrosurgery over laser are that no safety glasses are required and large amounts of tissue can be quickly removed ${ }^{[10,15]}$.

However, the disadvantages associated with electrosurgery included unpleasant odour, possibility of tissue damage or necrosis due to generation of lateral heat and gingival recession. It is contraindicated in patients with pace maker and procedures involving proximity to bone such as flap operations, mucogingival surgeries ${ }^{[14]}$.

Studies comparing the healing after scalpel and electrosurgical excision of soft tissues have demonstrated that lateral heat generated during electrosurgical procedures adversely affected the wound healing, thus delaying the healing process $[14,15]$. Clinically, satisfactory healing was observed by the end of the first postoperative month, in all the four cases treated with electrosurgery.

When electrosurgery is performed, the main objective is to produce a clean incision and/or coagulation with minimal lateral heat ${ }^{[14]}$. Kalkwarf et al. suggested that moving the electrode through tissue at a rate of seven $\mathrm{mm} / \mathrm{second}$ was compatible with good clinical technique and minimal production of lateral heat. Also, a cooling period of 8 seconds should be allowed between successive incisions with a needle electrode at the same surgical site. The period must be increased to fifteen seconds when a loop electrode is utilized for excisional procedures ${ }^{[16,17]}$.

Even though the procedure of lingual frenectomy is simple, the anatomical location and topography of the lingual tissue make it vulnerable to various intraoperative and postoperative complications. Postoperative complications of lingual frenectomy may include excessive hemorrhage, formation of mucus retention cyst or ranula, sublingual hematoma formation, development of sublingual and submandibular space infection, reattachment or recurrence of frenal attachment, development of new speech disorder or worsening of existing speech disorder and numbness or paresthesia of the tongue and neighbouring soft tissues ${ }^{[8,10]}$. However, no complication was reported in any of the four patients treated for ankyloglossia.

To achieve satisfactory results, postsurgical tongue training and speech therapy are strictly required. According to Tsaousoglou et al. ${ }^{[10]}$, the recommended tongue exercises postoperatively are as follows: Push the tongue in and out of mouth, open the mouth as wide as possible and attempt to touch the tip of the tongue to the back of the maxillary teeth, move the tongue from one side of the mouth to the other without moving the jaw, place food of choice on one side of mouth between the cheek and back teeth and then using the tongue, move the food from one side of the mouth to the other and then back again, pronounce consonant sounds of "f", " $v$ ", "d", "n", "l", "sh", "s", “zh", "r", and "z". All patients were advised to undergo tongue-training immediately after surgery 
and continue for 3 or 4 weeks postoperatively. Thereafter, the patients were evaluated for one month postoperative followup, advised to return for regular recalls and then referred to speech therapist for correction of speech defect. Significant improvement in speech was observed in all four treated patients.

\section{Conclusion}

Ankyloglossia limits the range of motion of the tongue, impairing its ability to fulfil its functions such as speech, position of teeth, swallowing, and certain social activities thereby affecting the self-esteem and confidence and leading to emotional and behavioural problems in children as well as adults. The cases presented in this paper were treated with frenectomy using electrosurgery followed by speech therapy, which resulted in favourable treatment outcome and patient satisfaction with improved tongue movements and correction of speech problems.

\section{References}

1. Messner AH, Lalakea ML, Aby J, Macmahon J, Bair E. Ankyloglossia: incidence and associated feeding difficulties. Arch Otolaryngol Head Neck Surg 2000;126(1):36-9.

2. Kotlow LA. Ankyloglossia (Tongue tie): A diagnostic and treatment quandary. Quintessence Int 1999;30:25962.

3. Chaubal TV, Dixit MB. Ankyloglossia and its management. J Indian SocPeriodontol 2011;15(3):270-2.

4. Ballard JL, Auer CE, Khoury JC. Ankyloglossia: assessment, incidence and effect of frenuloplasty on the breast-feeding dyad. Pediatrics, 2002, 110-e63.

5. Garcia Pola MJ, Gonzalez Garcia M, Garcia Martin JM, Gallas M, Seoane Leston J. A study of pathology associated with short lingual frenum. J Dent Child 2002;69:59-62.

6. Tuli A, Singh A. Monopolar diathermy used for correction of ankyloglossia. J Indian Soc Pedod Prev Dent 2010;28:130-3.

7. Dezio M, Piras A, Gallottini I, Denotti G. Tongue-tie, from embryology to treatment: a literature review. J PediatrNeonat Individual Med 2015;4(1):e40101.

8. Varadan M, Chopra A, Sanghavi AD, Sivaraman K, Gupta K. Etiology and clinical recommendations to manage the complications following lingual frenectomy: A critical review. J Stomatol Oral MaxillofacSurg 2019, $1-5$.

9. Reddy NR, Marudhappan Y, Devi R, Narang S. Clipping the (tongue) tie. J Indian SocPeriodontol 2014;18(3):3958 .

10. Tsaousoglou P, Topouzelis N, Vouros I, Sculean A. Diagnosis and treatment of ankyloglossia: A narrative review and a report of three cases. Quintessence Int 2016;47(6):523-34.

11. Messner AH, Lalakea ML. The effect of ankyloglossia on speech in children. Otolaryngol Head Neck Surg 2002;127:539-45.

12. Messner AH, Lalakea ML. Ankyloglossia: Controversies in management. Int $\mathbf{J}$ PediatrOtorhinolaryngol 2000;54:123-31.

13. Osman FS. Dental electrosurgery: General precautions. Can Dent Assoc J 1982;48:642.

14. Krejci RF. Kalkwarf KL, Krause-Hohenstein U. Electrosurgery- a biological approach. J ClinPeriodontol 1987; 14:557-63.
15. Morosolli AR, Veeck EB, Niccoli-Filho W, Gomes MF, das Gracas V, Goulart M, et al. Healing process after surgical treatment with scalpel, electrocautery and laser radiation: histomorphologic and histomorphometric analysis. Lasers Med Sci 2010;25:93-100.

16. Kalkwarf KL, Krejci RF, Edison AR, Reinhardt RA. Subjacent heat production during tissue excision with electrosurgery. Journal of Oral and Maxillofacial Surgery 1983a;41:653-57.

17. Kalkwarf KL, Krejci RF, Edison AR, Reinhardt RA. Lateral heat production secondary to electrosurgery incisions. Oral Surgery, Oral Medicine and Oral Pathology 1983b;55:344-48. 